\title{
Nanostructured Alkali-Metal Vapor Cells
}

\author{
T.F. Cutler $\odot,{ }^{1,}{ }^{*}$ W.J. Hamlyn, ${ }^{1}$ J. Renger, ${ }^{2}$ K.A. Whittaker, ${ }^{1}$ D. Pizzey $\odot,{ }^{1}$ I.G. Hughes $\odot,{ }^{1}$ \\ V. Sandoghdar ${ }^{2}, 2,3, \dagger$ and C.S. Adams ${ }^{1, *}$ \\ ${ }^{1}$ Joint Quantum Centre (JQC) Durham-Newcastle, Department of Physics, Durham University, \\ Rochester Building, South Road, Durham DH1 3LE, United Kingdom \\ ${ }^{2}$ Max Planck Institute for the Science of Light, Erlangen 91058, Germany \\ ${ }^{3}$ Friedrich Alexander University Erlangen-Nuremberg, Erlangen D-91058, Germany
}

(Received 21 April 2020; revised 21 July 2020; accepted 19 August 2020; published 21 September 2020)

\begin{abstract}
Atom-light interactions in micro- and nanoscale systems hold great promise for alternative technologies based on integrated emitters and optical modes. We present the design architecture, construction method, and characterization of an all-glass alkali-metal vapor cell with nanometer-scale internal structure. Our cell has a glue-free design that allows versatile optical access, in particular with high numerical aperture optics, and incorporates a compact integrated heating system in the form of an external deposited indium tin oxide layer. By performing spectroscopy in different illumination and detection schemes, we investigate atomic densities and velocity distributions in various nanoscopic landscapes. We apply a two-photon excitation scheme to atoms confined in one dimension within our cells, achieving resonance line widths more than an order of magnitude smaller than the Doppler width. We also demonstrate sub-Doppler line widths for atoms confined in two dimensions to micron-sized channels. Furthermore, we illustrate control over vapor density within our cells through nanoscale confinement alone, which could offer a scalable route towards room-temperature devices with single atoms within an interaction volume. Our design offers a robust platform for miniaturized devices that could easily be combined with integrated photonic circuits.
\end{abstract}

DOI: 10.1103/PhysRevApplied.14.034054

\section{INTRODUCTION}

Individual atoms or atomic ensembles offer an attractive platform for quantum sensing and devices. Atom-light interactions have already found use in applications such as clocks [1,2], optical filtering [3-5], magnetometry [6$15]$, and laser stabilization [16,17]. However, most atomic devices are either macroscopic or at best mesoscopic [9]. For some applications, it would be attractive to achieve nanoscale confinement or localization, which is standard for solid-state systems [18] but challenging for atoms.

Indeed, work towards confinement of atoms and light fields on the micro- and nanoscales has increasingly offered diverse access to studying fundamental physics [19-27], sensing [10,28-32], miniaturization of optical devices $[1,7,33]$, and quantum technologies [34-39]. Although the first atom-on-chip attempts date back about two decades

*thomas.cutler@durham.ac.uk

†vahid.sandoghdar@mpl.mpg.de

\$c.s.adams@durham.ac.uk

Published by the American Physical Society under the terms of the Creative Commons Attribution 4.0 International license. Further distribution of this work must maintain attribution to the author(s) and the published article's title, journal citation, and DOI.
[40,41], the difficulties of taming surface effects such as van der Waals forces [42-44] have rendered the control of cold atoms at nanoscopic distances elusive [45].

The continued study of atom-light interaction in thermal vapors has offered diverse access to fundamental physics [46-48], and miniaturization of such thermal vapor systems is an emerging alternative approach to atomic physics at the micrometer and nanometer scales. Examples include confinement of vapors to micron-sized hollow core fibers [49] and construction of submicron alkalimetal vapor cells [32,50-52]. Developments in miniaturized alkali-metal vapor cell technology on the millimeter scale have already enabled alternative atom-based devices, such as chip-scale atomic clocks [53] and magnetometers [7]. Promising further technological developments on the micrometer scale include integrated diffractive elements [54]. As well as the fundamental interest in atom-light interactions at the nanoscale already highlighted, there is also interest in interfacing thermal vapors with nanophotonics [55-58] and integrated opto-mechanical features [59]. However, much work remains to be done before nanometer-scale vapor-based devices can be used in technological applications, e.g., for local sensing [15] or quantum networks $[36,60]$. In particular, reliable fabrication methods are needed for the realization of scalable and efficient platforms. Furthermore, the interactions of the 
atoms with the cell walls $[20,29,30,42,61]$ as well as their velocity distribution and diffusion behavior have to be investigated and understood.

In this work we present a platform that produces long lifetime (at least one year) alkali-metal vapor nanocells. Using laser lithography and reactive ion etching, we create regions with internal dimensions from $2 \mu \mathrm{m}$ to $400 \mathrm{~nm}$ (though smaller structures with dimension below $100 \mathrm{~nm}$ are possible [62]), filled with rubidium vapor. Note that in this work we will use "nano" to describe the submicron length scales present in our cells. We demonstrate full optical access that facilitates the use of conventional high numerical aperture (NA) optics for efficient atomlight coupling. Previously reported nanocells are generally wedge shaped, with confinement in one spatial dimension decreasing continuously to $0 \mu \mathrm{m}[32,50]$. This wedge design, in combination with thick cell windows, does not allow for such versatile optical access or use of high-NA optics, nor near-arbitrary patterned confinement geometries. In contrast, existing works demonstrate the use of hollow-core fibers for confinement of atomic vapors in not one but two spatial dimensions. However, these are limited to core sizes of the order of several microns. In this study, we show that our nanocell etching process presents a significant improvement, producing atom confinement in one and two dimensions with submicron length scales. For the reasons outlined above, our design and manufacturing process presents a significant advancement in nanocell and confined-vapor technology. We illustrate the benefits and capabilities of our nanocell by experimentally demonstrating two excitation and detection schemes for atoms confined within various nanoscale structures. Our approach opens doors to efficient and compact architectures for performing quantum nano-optical studies.

\section{NANOCELL FABRICATION}

In Fig. 1(a) we display the schematics of our glass nanocells, depicting a side view of the cell region, with nanoscopic features located at positions (iii) and (iv). A tube conduit [diameter $5 \mathrm{~mm}$, labeled (vi)] connects this nanostructured region to an alkali-metal reservoir. The glass cell is assembled from three distinct parts that we refer to as the cover slide [labeled (i)], the block [labeled (ii)], and the reservoir [located below label (vi)]. In this work, the block is a fused silica cuboid of dimensions $10 \times 20 \times 30 \mathrm{~mm}^{3}$, though this is chosen for manufacturing convenience and could be considerably reduced in size for future applications. The cover slide is a fused silica panel of dimensions $0.5 \times 20 \times 30 \mathrm{~mm}^{3}$ into which we "write" the nanostructures of choice. To do this, we pattern micro- and nanoscale features into photoresist using direct-write laser lithography, and transfer these to the glass substrate using reactive ion etching [62]. In Fig. 1(b)
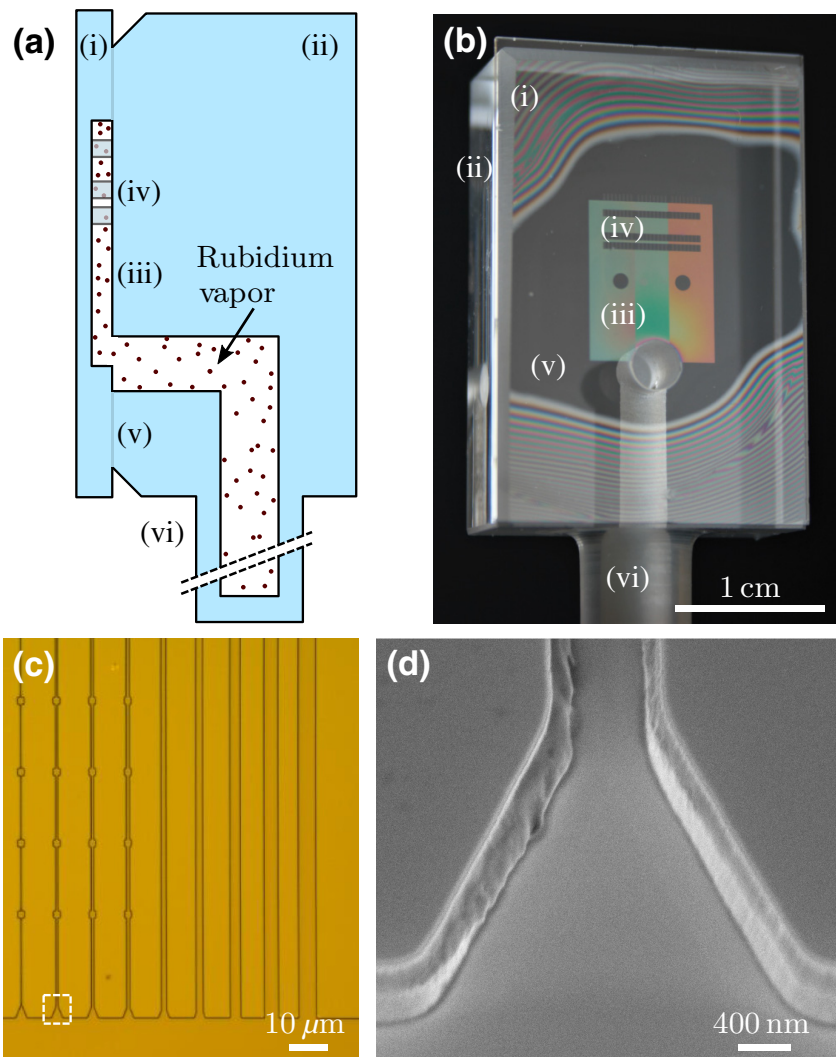

FIG. 1. (a) Side cross-section schematic (not to scale) and (b) front-facing photograph of the nanocell. The etched cover slide (i) is contacted onto the block (ii) such that the etched patterns form a nanothickness region (iii) and nanochannels (iv) between the two glass pieces. An optical contact bond (v) surrounds the etched area to act as a vacuum seal. The rubidium reservoir below (vi) is attached to provide atomic vapor into the nanostructured region. This cell contains areas with confinement in both one and two dimensions. (c) Front-facing bright field microscopy images of various channel structures, with widths 400-5000 nm. A number of copies of these structures are etched into the cover slide to provide a range of depths (400-2000 nm). These channel structures reside in the horizontal black bars, labeled (iv) in (b). Vapor is supplied from below in this image. (d) SEM image of a single channel aperture [indicated by the white box in (c)].

we show a photograph of the cell under white-light illumination (full details of cell characterization is discussed in Sec. III).

The block is optically polished on all sides to increase optical access to the nanoscopic regions, and to facilitate the formation of an optical contact bond (OCB). We form an OCB between the cover slide and the block to act as a vacuum seal encircling the patterned area. The cover slide is placed on the block such that the micro- and nanopatterns form a confined region between the two pieces, which also overlaps the internal tube of the block to allow vapor to enter. In Fig. 1(b) we show the OCB surrounding the patterned area, seen as the lack of Newton's fringes between 

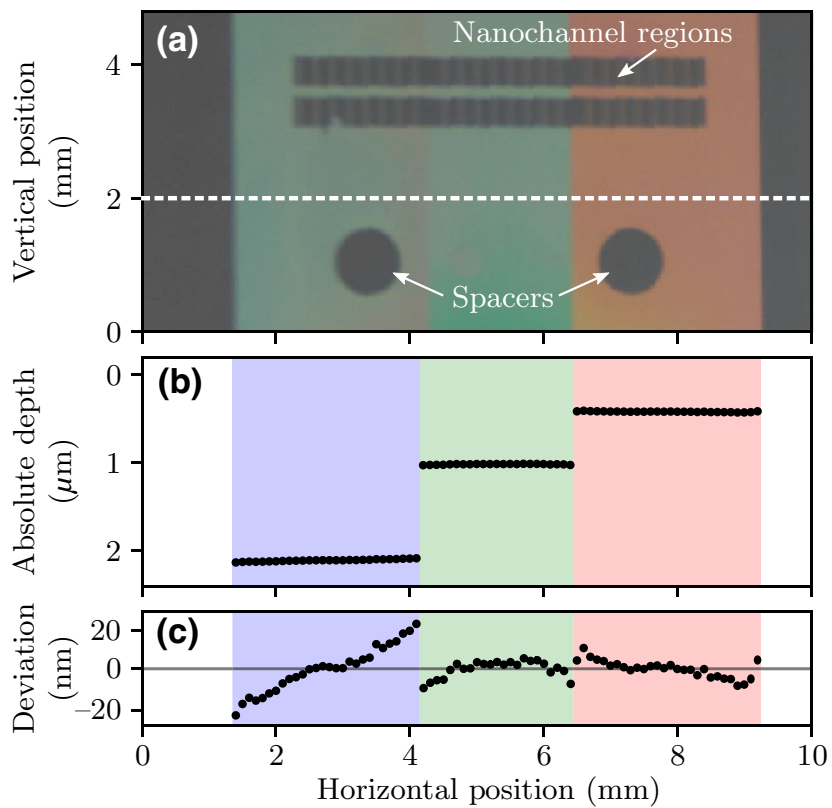

FIG. 2. (a) A close section of Fig. 1(b), showing three discrete thickness regions. The "spacers" are columns of fused silica left unetched to prevent the cavity from bowing during evacuation. The horizontal features above are where the nanochannels are located. The dashed white line indicates the axis along which measurements in (b) are recorded. (b) Absolute cavity thickness (averaged over the $100 \mu \mathrm{m}$ focal spot size) extracted from the fitted Fabry-Pérot transmission model as a function of lateral position across the nanocell. The uncertainty in each depth is of order $1 \mathrm{~nm}$ and is thus too small to be seen. (c) The deviation of each data point from the mean value for each of the three colored sections.

the two fused silica pieces. Once an OCB encircling the nanoregions and milled aperture has been formed, the bond is made permanent by firing in a kiln at $1000^{\circ} \mathrm{C}$ for $6 \mathrm{~h}$ at atmospheric pressure. The OCB process up until firing is reversible. Two fused silica spacer columns [labeled in Fig. 2(a)] are included in our cell design to prevent the cavity thickness from deviating from the design specification due to bowing during the next fabrication step, when the air is pumped out to produce a vacuum.

To load the nanocell with alkali-metal atoms (in our case rubidium at natural abundance), the fired pieces are attached to a borosilicate glass manifold by glassblowing. A vacuum pump and a rubidium break seal ampoule are attached to the manifold, which is evacuated and generally sealed off at $10^{-6}$ mbar. The rubidium ampoule is broken and, by melting with a low flame, liquid rubidium is made to flow towards the block. A short (typically $5 \mathrm{~cm}$ ) section of the manifold immediately adjacent to the block, and containing condensed rubidium, is then sealed and detached to enclose the complete nanocell. This section of borosilicate glass tube attached to the bottom of the block is referred to as the reservoir [see Fig. 1(a), part (vi)]. Note that the cell is not baked during the evacuation process.
However, we expect that the prebaking at $1000^{\circ} \mathrm{C}$ similarly acts to remove surface adsorbates, and spectroscopic measurements (such as those shown later in this study) do not show prohibitive resonance line shifts or broadening, and thus we do not consider residual background gas to be a problem.

We find that our glue-free design is very robust, and we have produced numerous nanocells with an operation lifetime of over $1 \mathrm{yr}$. As is standard for thermal vapor experiments, our cells are heated to vary the internal vapor density. Our cells can withstand dozens of heating and cooling cycles between room temperature and $180^{\circ} \mathrm{C}$. In typical operation both the cell block and the reservoir are independently heated, so as to maintain a thermal gradient between the two and thus avoid rubidium condensation in the nanostructured regions.

Heating is achieved via indium tin oxide (ITO) regions on external surfaces of the cell, which act as resistive heaters when an electric current is applied [not shown in Fig. 1(b)], whilst also being transparent at our wavelengths of interest. Generally, a $15 \mathrm{~mm}$ square patch of ITO is deposited onto the back surface of the cell block to a thickness of $400 \mathrm{~nm}$. The ITO is deposited by sputtering using an Ar plasma, and annealed in air at $450^{\circ} \mathrm{C}$. The ITO is contacted on two sides by rectangular $15 \times 4 \mathrm{~mm}^{2}$ regions made up of a Ti adhesion layer and an Au layer, which act as electrical contacts. The resulting integrated heater has a resistance of the order $50 \Omega$. The process can then be repeated on the cell reservoir so that this can be heated independently of the cell block and nanoregions (see the Supplemental Material [63] for more detail on the ITO heating solution). Experiments with thermal vapors often rely on bulky ovens for cell heating, but the compactness and transparency of our ITO layer allows heating of the cell without compromising optical access or the experimental footprint.

To populate the nanoscale regions with atoms, we find that it is favorable to periodically "flood" these areas with liquid rubidium by inducing a reverse temperature gradient (reservoir hotter than the nanoregion) until condensed rubidium is visibly present, and then returning to a conventional temperature gradient to evacuate the nanoregions again. After such a cycle, deposits of rubidium remain on the inner cell surfaces that act as local reservoirs. Such cycles are generally only necessary after timescales of the order of multiple weeks, where the cell has been heated and in operation daily.

\section{CHARACTERIZATION}

In Fig. 1(b) we show a completed nanocell with the patterned regions made visible by white-light interference in the void formed between the two fused silica pieces, producing strong colors. More subtle, however, is the fact that the colors change both gradually and in discrete steps 
across the patterned area of the nanocell. The discrete color changes occur by design due to the discrete depth changes selected when patterning the cover slide. The gradual color changes are a result of the surface of the block and the internal surface of the cover slide being nonparallel. We also note that the gradients generally differ before and after the kiln firing process, suggesting that the process induces changes in mechanical stress in the two fused silica pieces.

To characterize the cells, the thickness of the patterned regions is measured after the assembly is complete. To do so, we exploit the fact that the nanothickness region acts as a low finesse Fabry-Pérot cavity, with the internal surfaces of the fused silica cavity acting as low reflectivity mirrors. The transmission, $T$, of a Fabry-Pérot interferometer is [64]

$$
T=\frac{(1-R)^{2}}{1-2 R \cos \delta+R^{2}},
$$

where $R$ is the reflectivity at the fused silica interface and $\delta=4 \pi l / \lambda$ is the phase shift of each subsequent round-trip reflection in the cavity, with $l$ the cavity length and $\lambda$ the wavelength of the probing light. The free spectral range of our microcavities is of order $\lambda$; hence, we use white light to span multiple Fabry-Pérot transmission peaks, which are fitted with a model using Eq. (1) (see the Supplemental Material [63] for further detail).

In Fig. 2(b) we show the result of a horizontal sweep across the face of the nanocell, with white-light spectra taken at $0.1 \mathrm{~mm}$ intervals along the axis marked (dashed white line) in Fig. 2(a). This method gives high precision measurements of cavity thickness, averaged over the $100 \mu \mathrm{m}$ focal spot size, at each point (with error bars of order $1 \mathrm{~nm}$ ). From left to right, we find mean values of 2070, 1020, and $420 \mathrm{~nm}$ for the discrete thickness regions. The deviation from these mean values, shown in panel (c), reveals a gradual thickness change across the cell. The variations within each thickness region are of the order of $1 \%$, and show that the nanocell is slightly concave, which is likely an effect of inhomogeneous reactive ion etching.

The white-light technique gives information on average thickness trends across the extent of the nanocell. However, due to the area averaged over for each data point, this technique does not give information on surface roughness or defects on smaller length scales than the focal spot size. By using atomic force microscopy (AFM) on similarly prepared substrates, we find that a rms roughness below $1 \mathrm{~nm}$ is routinely achievable after etching. An example is shown in Fig. 3 for a surface etched to a depth of $500 \mathrm{~nm}$. Both the $5 \times 5 \mu \mathrm{m}^{2}$ region shown in (a) and the example horizontal profile shown in (b) clearly illustrate that the etched surfaces are of good optical quality. The exact roughness of each sample varies, dependent on the etch depth and conditions, and small particles or holes of order $10 \mathrm{~nm}$ in size may remain. However, as we have shown in this section, it is possible to control and characterize our surfaces on

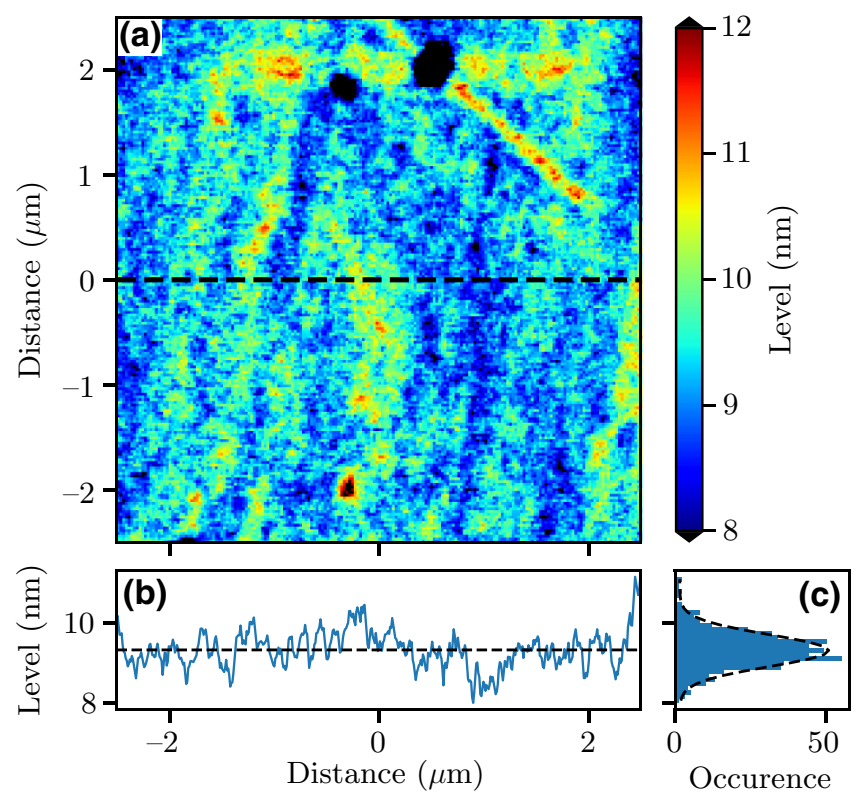

FIG. 3. (a) Image produced using AFM showing the surface roughness of a fused silica substrate etched to a depth of $500 \mathrm{~nm}$. This surface is prepared in the same way as the nanocells used in this work. This measurement is performed before the cell bonding and baking steps. The rms roughness is $0.7 \mathrm{~nm}$. If the defect at the top of the image is excluded then this becomes $0.6 \mathrm{~nm}$. For comparison, an unetched surface of the same fused silica used (not shown) gave a rms roughness of $0.43 \mathrm{~nm}$. (b) A horizontal cut showing the surface profile along the dashed line shown in (a). The average level is indicated (black dashed line). (c) The distribution of points from (b) relative to the average surface level, with a Gaussian fit (black dashed line).

various length scales in order to understand the residual roughness, particles, and gradients that may exist.

\section{DETECTION SCHEMES AND RESULTS}

The topic of detection schemes in thin cells has already received significant attention, with standard examples including transmission and selective reflection spectroscopy (see, for example, Refs. [28,50,67]), the latter having grown in popularity since the pioneering work of Woerdman and Shuurmans in probing atoms close to surfaces in 1975 [68]. In this section, we begin by detailing the two detection schemes used in this work. These schemes exemplify the versatility of our vapor cell design to different input beam geometries, in particular the flexibility of optical access enabled the cell geometry and integrated heater.

The energy levels of rubidium relevant to the detection methods used in this work are shown in Fig. 4. In the most straightforward scheme, a single tunable laser at $\lambda=780 \mathrm{~nm}$ is used to excite $\mathrm{Rb}$ atoms from their ground state $5 \mathrm{~S}_{1 / 2}$ to the excited state $5 \mathrm{P}_{3 / 2}$, i.e., on the $\mathrm{D} 2$ line. Extinction spectroscopy in transmission on this (or the 


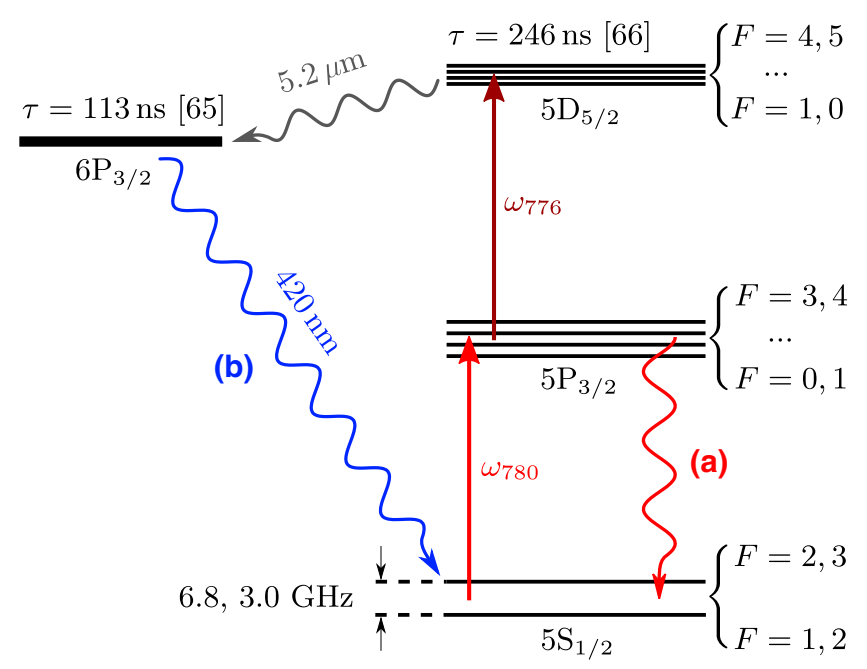

FIG. 4. Partial rubidium energy level diagram showing key transitions and detunings used to probe the atoms inside the nanocell in this work. Relevant hyperfine states are indicated for ${ }^{87} \mathrm{Rb},{ }^{85} \mathrm{Rb}$ (note that these are omitted for $6 \mathrm{P}_{3 / 2}$, which we do not spectroscopically probe). Two detection schemes used in this work are labeled (a),(b). Scheme (a) relies on a single excitation laser at $780 \mathrm{~nm}$ addressing the rubidium D2 line, driving population to the $5 \mathrm{P}_{3 / 2}$ state that then decays via emission of a photon of the same frequency. Scheme (b) employs two lasers at 780 and $776 \mathrm{~nm}$ to drive atoms to the $5 \mathrm{D}_{5 / 2}$ state, from which they can decay via the $6 \mathrm{P}_{3 / 2}$ state and produce a photon at $420 \mathrm{~nm}$. The lifetimes of each of these states, $\tau$, are indicated.

D1 line, not shown in Fig. 4) has been the most widely adopted detection technique for studying thermal vapors due to the simplicity of implementation. However, when the optical depth of the system is decreased, as in the case of a nanothickness vapor, it becomes more challenging to detect the extinction signal due to a reduced signal-to-noise ratio [69]. Our geometry allows us to instead perform fluorescence measurements efficiently, employing a high-NA objective lens to collect atomic fluorescence through the thin cover glass.

Two fluorescence-based detection schemes are used in this work. A single-photon scheme-labeled (a) in Fig. 4 is implemented using a total internal reflection fluorescence (TIRF) geometry (see Fig. 5). However, as this scheme is prone to scattering, an alternative two-photon excitation scheme is also developed in order to study atoms in micron and submicron channels. In this scheme a $776 \mathrm{~nm}$ laser is added to drive population to the $5 \mathrm{D}_{5 / 2}$ state, from which there is approximately $7.5 \%$ probability that an atom will decay via the $6 \mathrm{P}_{3 / 2}$ state and produce a $420 \mathrm{~nm}$ photon [13] [labeled (b) in Fig. 4]. This light can be spectrally separated from scattered excitation laser light, providing an even higher signal-to-background ratio than in the single-photon scheme.

Below we discuss the two detection schemes in more detail, and employ them to record spectroscopic data from

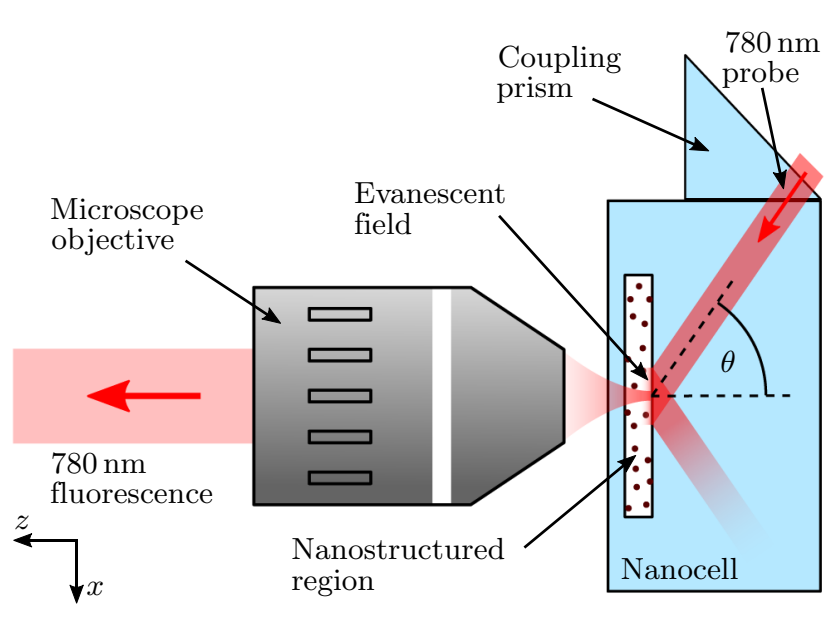

FIG. 5. A top-down schematic diagram of the TIRF setup. A coupling prism is contacted to the cell with index-matching oil, and this is used to deliver a $780 \mathrm{~nm}$ probe beam into the nanocell at an angle $\theta$ to the normal. When incident on the back interface between the nanoregion and the glass, the beam totally internally reflects and an evanescent field is formed inside the nanoregion. This field excites the atomic vapor. Fluorescence is collected through the front face of the cell via an $f=2 \mathrm{~mm}$ focal length, $\mathrm{NA}=0.7$ plan apochromat objective lens, onto a single-photon avalanche diode. Note that the coupling prism used is the same for all datasets, and simply improves ease of alignment at more extreme angles.

atoms confined to the nanocell. In all cases the frequency axis is calibrated by a reference $\mathrm{Rb}$ cell in a methodology similar to that performed in Ref. [70], where the zero of detuning is chosen to be the weighted line center of the rubidium $\mathrm{D} 2$ transition.

\section{A. Total internal reflection fluorescence}

A schematic of the TIRF setup is shown in Fig. 5. Total internal reflection occurs at the glass-vapor interface, and a single-color evanescent light field is created that can drive atomic excitation and induce fluorescence. Although strictly three dimensional, we consider only the variation of the laser field along the axial distance $z$ from the interface between the fused silica wall and the vapor. Hence, the evanescent field has the general form $I(z)=I_{0} e^{-z / d}$, where $I_{0}$ is the intensity at the boundary immediately outside of the medium in which the total internal reflection is occurring, $z$ is the perpendicular distance from the fused silica wall into the vapor, and $d$ is the evanescent field $1 / e$ decay length. This is given by [71]

$$
d=\frac{\lambda}{4 \pi}\left(n_{1}^{2} \sin ^{2} \theta-n_{2}^{2}\right)^{-1 / 2},
$$

with $n_{1}$ the refractive index of fused silica (approximately equal to 1.45), $n_{2}$ the refractive index of the vapor (nominally taken as 1), $\theta$ the incident angle, and $\lambda$ the vacuum wavelength of the light field (we probe the D2 line 
at $\lambda=780 \mathrm{~nm}$ ). Typically, we have $d<\lambda / 2 \pi$, and thus expect frustrated total internal reflection to have little effect in our nanocells with internal dimensions of the order $\lambda$.

To demonstrate the TIRF methodology, experiments are performed to study the transit-time broadening induced by varying evanescent field decay lengths. The nanocell is heated to $100^{\circ} \mathrm{C}$, and fluorescence counts are collected for $10 \mathrm{~s}$ with a probe beam power of $10 \mu \mathrm{W}$. The resulting fluorescence spectra shown in Fig. 6 contain transitions from each of the two hyperfine $5 \mathrm{~S}_{1 / 2}$ ground states, for each of the two naturally occurring rubidium isotopes, to the $5 \mathrm{P}_{3 / 2}$ state (see Fig. 4) [70]. The hyperfine structure of the $5 \mathrm{P}_{3 / 2}$ state, with features on the scale of $100 \mathrm{MHz}$, is not resolved due to Doppler broadening.
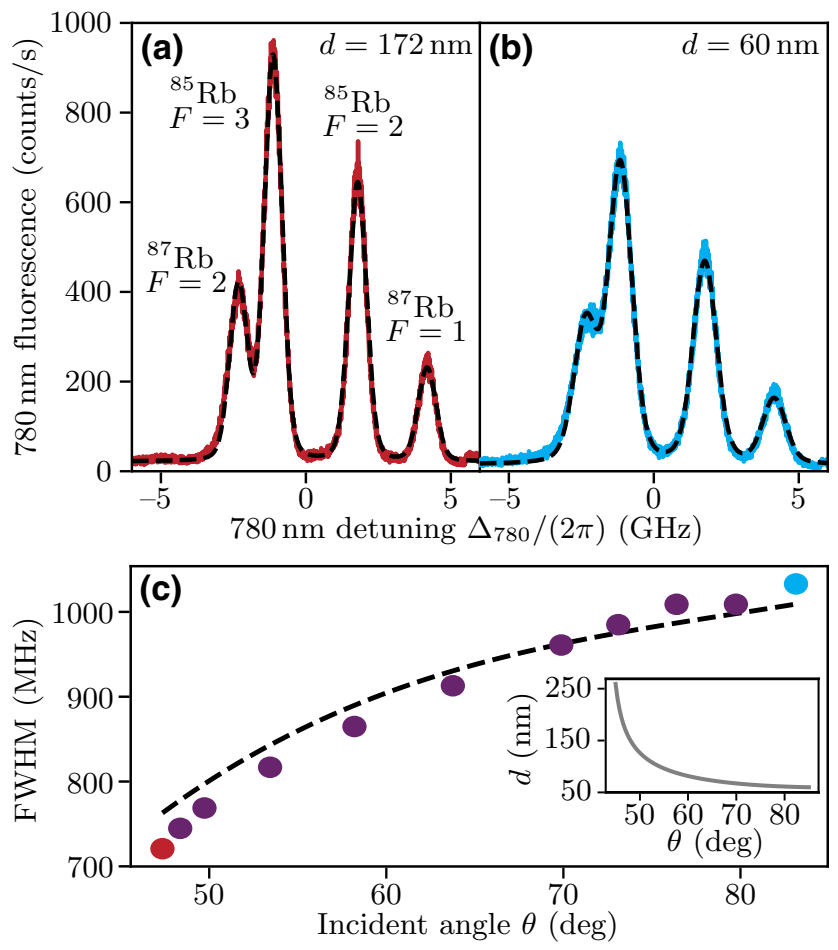

FIG. 6. (a) A TIRF spectrum (red) recorded with incident angle $\theta=47^{\circ}$ onto a $2 \mu \mathrm{m}$ thick vapor layer, with fitted Voigt model (black dashes). The spectrum has narrow line widths due to the long evanescent field depth $(d=172 \mathrm{~nm})$ resulting in less transit-time broadening. The hyperfine ground states corresponding to each resonance have been labeled for clarity. (b) TIRF spectrum (blue) as in (a), but with $\theta=83^{\circ}$, resulting in a shorter evanescent field $(d=60 \mathrm{~nm})$ and thus greater transit-time broadening. Fitted model shown as black dashed line. (c) Measured FWHM extracted from the Voigt model for TIRF spectra as a function of $\theta$ (colored circles). Horizontal error bars cannot be seen but are of order $1^{\circ}$. The extreme data points are color matched to the spectra in (a),(b). Also plotted is the result of a Monte Carlo simulation of thermal atoms traveling within the detection volume (black dashed line). Inset shows the evanescent decay length as a function of $\theta$ [see Eq. (2)].
To analyze the observed spectra, we consider a lineshape model made by the summing of four Voigt profiles, each centered on one of the four hyperfine ground states observed in the fluorescence spectrum. The full width at half maximum (FWHM) of one Voigt profile is plotted as a function of $\theta$ [colored circles in Fig. 6(c)]. The trend reveals that the spectra become broader as the incident beam angle is increased. We understand this to be a transit-time broadening caused by the reduced characteristic decay length, $d$, of the evanescent light field that probes the atoms [see Fig. 6(c) inset].

To examine the observed line-width behavior further, we performed a Monte Carlo simulation. The simulation initializes 3000 individual atoms with a random velocity vector $\mathbf{v}=v_{x} \hat{x}+v_{y} \hat{y}+v_{z} \hat{z}$ (see Fig. 5 for axes definition) such that the distribution of velocity vectors approximates the Maxwell-Boltzmann distribution as expected for an ideal thermal vapor. The spectrum of the ensemble is made up of Lorentzian line shapes contributed by individual atoms, which are modified in two ways. First, the line center is shifted by the Doppler effect $\mathbf{k} \cdot \mathbf{v}=k v_{z}$ as the atom moves with respect to the laboratory frame. Second, the width of the Lorentzian is modified by the short transit time $t_{\text {trans }}=d / v_{z}$ across the short decay length $d$ of the evanescent field along $z$. To put this timescale in perspective, we note that at a typical temperature of $100{ }^{\circ} \mathrm{C}$ a thermal atom has a mean speed of $330 \mathrm{~m} \mathrm{~s}^{-1}$, corresponding to $t_{\text {trans }}=0.3 \mathrm{~ns}$ across a distance $d=100 \mathrm{~nm}$. We define an effective line width $\Gamma_{\text {eff }}$ per atom using

$$
\Gamma_{\mathrm{eff}}^{2}=\Gamma_{\mathrm{nat}}^{2}+\left(\frac{\alpha}{t_{\text {trans }}}\right)^{2}
$$

where $\Gamma_{\text {nat }}$ is the natural line width $(2 \pi \times 6 \mathrm{MHz}$ [72] $)$ and $\alpha$ is a unitless factor that attenuates the transit-time broadening. We do not consider atomic interactions or collisions, as typically we have atomic densities of order $10^{12} \mathrm{~cm}^{-3}$ and do not have buffer gas within our cells. The 3000 atom simulation is repeated ten times at a number of discrete values of incident beam angle $\theta$ to return a mean and standard error for each. A fit to the simulated data is plotted as the black dashed line in Fig. 6(c). We find that with $\alpha=0.5$ our simple simulation is sufficient to capture the experimentally observed trend. For comparison, for transit-time broadening of atoms traversing a Gaussian laser beam the comparable factor is $\alpha \approx 0.2$ [73]. We speculate that the differences between observation and theory come about due to the evanescent field gradient experienced by the atoms, as well as the reduced probability of a fast moving atom being excited by the laser field. Neither of these effects are included in our model. The latter means that atoms with a low transit time are less likely to contribute to the fluorescence signal, reducing the overall broadening effect. 
In this section we have demonstrated the TIRF methodology in our nanocells, and how this can be used to probe physical effects on the nanoscale. We have also demonstrated that simple modeling can reproduce the experimentally observed trends in line shapes with varying evanescent decay lengths. As illustrated in Figs. 6(a) and 6(b), the TIRF method gives high signal-tobackground ratios for spectroscopy, with far off-resonance background noise two orders of magnitude lower than onresonance fluorescence count rates. However, this singlephoton method relies on detection of fluorescence photons at the same frequency as the input laser photons, so although it is theoretically a dark-field method, it is in practice not free of background scattering. This poses a problem in particular for cells with more complex structures such as one-dimensional nanochannels [see, for example, Fig. 1(c)], where laser light is efficiently scattered by the edges. In the following section we detail a different excitation scheme and geometry accessible using our nanocells, which allows high signal-to-background ratios for spectroscopy even in these regimes.

\section{B. Two-photon fluorescence microscopy}

In this section, we detail a two-photon excitation scheme that allows fluorescence detection at $420 \mathrm{~nm}$, and demonstrate its use for probing atoms confined in one and two dimensions within our nanocells. The scheme is depicted in Fig. 4, with the fluorescence pathway used for detection labeled (b). This scheme allows for straightforward filtering of any excitation photons scattered by the substrate, using commercially available bandpass filters.

Our nanocell design allows for the use of high-NA optics, which allows us to optically resolve nanoscale structures, to address and collect fluorescence from atoms within individual structures, and to achieve extremely high Rabi frequencies even on weak transitions. In this section we employ a NA $=0.7$ plan apochromat microscope objective to address atoms within volumes approximately $\lambda^{3}$ confined in nanoscale structures, where spatial confinement is typically less than or equal to $1 \mu \mathrm{m}$ in one or two dimensions. In Fig. 7 we show the experimental setup, where the two excitation lasers are delivered to the nanoregions in co- or counterpropagating arrangements, and atomic fluorescence photons are collected via the microscope objective.

Our high-NA approach leads to small beam sizes and extremely high Rabi frequencies at modest laser power. For example, a $776 \mathrm{~nm}$ laser power of $20 \mu \mathrm{W}$ and a spot size of 1 micron gives a Rabi frequency of $2.9 \mathrm{GHz}$ on the $5 \mathrm{P}_{3 / 2} \rightarrow 5 \mathrm{D}_{5 / 2}$ transition (using the dipole matrix element measured for this transition in Ref. [74]). This means that atoms in the $5 \mathrm{P}_{3 / 2}$ state are promoted to the $5 \mathrm{D}_{5 / 2}$ state at a rate much faster than the Doppler width. Below we discuss

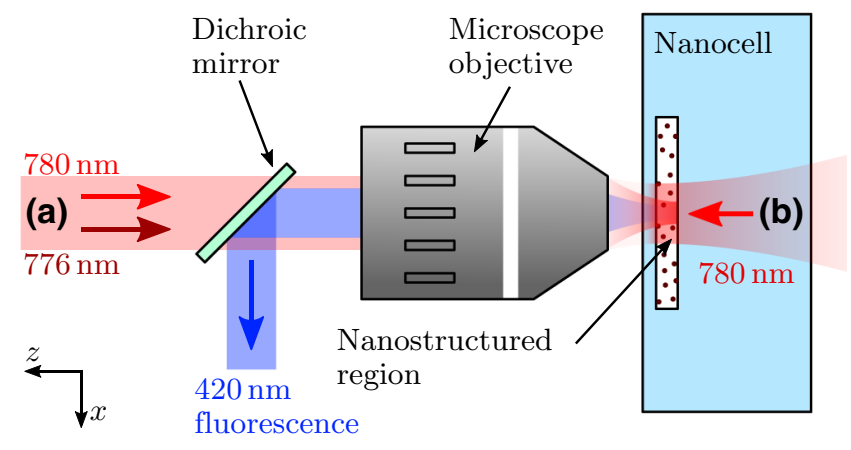

FIG. 7. Top-down schematic of the fundamental components in the two-photon fluorescence microscopy (TPFM) setup. Probe lasers at 780 and $776 \mathrm{~nm}$ are delivered to the nanoregions of the vapor cell, with the $780 \mathrm{~nm}$ beam copropagating (a) or counterpropagating (b). Beams along pathway (a) are focused into the nanocell by a $100 \times$ plan apochromat microscope objective, with $2 \mathrm{~mm}$ focal length, $6 \mathrm{~mm}$ working distance, and $\mathrm{NA}=0.7$. Along pathway (b) the beam is weakly focused through the back face of the cell using an $f=100 \mathrm{~mm}$ lens. Atoms inside the nanostructures produce $420 \mathrm{~nm}$ fluorescence (see the excitation scheme in Fig. 4), which is collected by the microscope objective, and reflected by a dichroic mirror onto a photon-counting photomultiplier tube (PMT) for detection.

the effect of this on resonance line shapes observed in both co- and counterpropagating regimes.

In the experiment, we scan the first step $780 \mathrm{~nm}$ laser through the D2 resonance while employing excited-state polarization spectroscopy [75] to frequency stabilize a second step laser at $776 \mathrm{~nm}$ to the $5 \mathrm{P}_{3 / 2} \rightarrow 5 \mathrm{D}_{5 / 2}$ transition. Subsequently, we detect $420 \mathrm{~nm}$ fluorescence photons from the $6 \mathrm{P}_{3 / 2} \rightarrow 5 \mathrm{~S}_{1 / 2}$ decay (see Fig. 4).

We first report on the results of implementing a TPFM scheme in which the two excitation beams are counterpropagating in the nanocell [see Fig. 7(b)]. As can be seen in Fig. 8, this scheme gives well-resolved sub-Doppler fluorescence resonances corresponding to the transitions from the hyperfine $5 \mathrm{~S}_{1 / 2}$ ground states to the allowed $5 \mathrm{P}_{3 / 2}$ hyperfine states. In this scheme, a large $780 \mathrm{~nm}$ excitation volume allows atoms to be excited to the $5 \mathrm{P}_{3 / 2}$ hyperfine states. Some of these excited atoms will subsequently enter the tightly focused $776 \mathrm{~nm}$ spot, and will then sequentially excite to the $5 \mathrm{D}_{5 / 2}$ state due to the high Rabi frequency as noted above. Hence, the splittings and line strengths observed correspond closely to those for the $5 \mathrm{P}_{3 / 2}$ hyperfine levels. A Voigt fit yields a FWHM of $(32 \pm 1) \mathrm{MHz}$, consistent with transit-time broadening with $1 \mu \mathrm{m}$ confinement. In contrast to single laser pump-probe schemes, crossover resonances are also absent, affording better resolution of the allowed hyperfine transitions. Note that nanocells are an attractive platform for the study of the interaction of atoms and high intensity laser fields, as the extent of the confined atoms can be smaller than the Rayleigh range, thus removing the necessity to average 


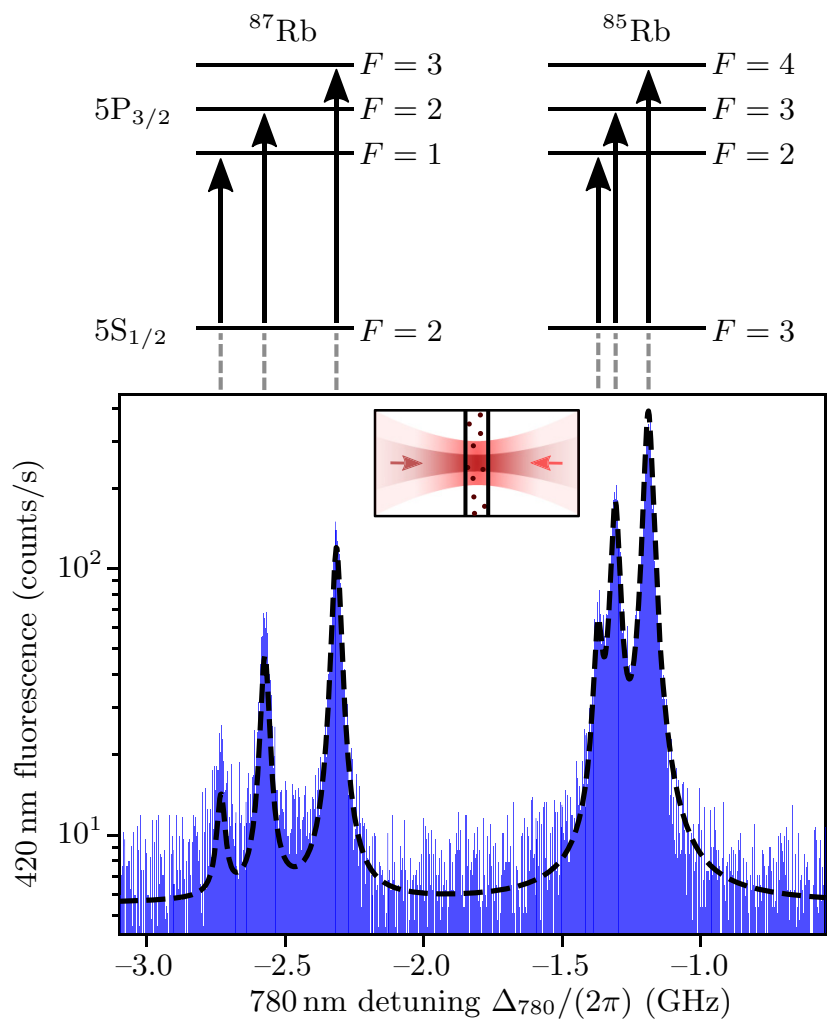

FIG. 8. The $420 \mathrm{~nm}$ fluorescence (blue histogram) recorded from a region of the nanocell with $1 \mu \mathrm{m}$ depth (i.e., confinement along $z$ ). A counterpropagating two-photon excitation geometry is used (see the inset diagram illustrating atomic layer and input beams, as well as Fig. 7). Note that this spectrum corresponds to the left half of that shown in Fig. 6(a). An empirical fit comprising six Voigt profiles (black dashed line) is performed, with the locations of the resonances constrained to the relevant hyperfine transition frequencies, their magnitudes constrained to the isotopic abundance-weighted transition strengths, and their widths set to be equal (but allowed to vary as a fit parameter). This yields a line width of $(32 \pm 1) \mathrm{MHz}$. Partial energy level diagrams are shown above for reference. For this dataset, powers of $350 \mu \mathrm{W}$ at $780 \mathrm{~nm}$ (focused with the $f=100 \mathrm{~mm}$ lens) and $20 \mu \mathrm{W}$ at $776 \mathrm{~nm}$ (focused with the $f=2 \mathrm{~mm}$ lens) are used, with a cell temperature of $60^{\circ} \mathrm{C}$ and an integration time of $12 \mathrm{~h}$.

over different intensity regimes (which would be the case in a standard millimeter-sized vapor cell). Our bespoke nanocell design allows high-NA objectives to be placed close to the atomic layer to facilitate such studies.

Spectra are also obtained using the TPFM method with a copropagating geometry [see Fig. 7(a)], and a selection of these are shown in Fig. 9. Here the nanocell is displaced along the optical axis of the microscope objective, such that atoms sampled the laser intensity profile locally. The spectral features in the datasets arise due to the hyperfine structure of the $5 \mathrm{P}_{3 / 2}$ state, though not all hyperfine states are fully observed or resolved. We no longer observe the same activity ratios between hyperfine levels as in the counterpropagating case (Fig. 8). We attribute this to the fact that, in this scheme, both lasers are tightly focused, leading to a small excitation volume. This means atoms must be excited to the $5 \mathrm{D}_{5 / 2}$ state within the timescale of transit across the $1 \mu \mathrm{m}$ spot (approximately $4 \mathrm{~ns}$ ). In contrast to the sequential excitation in the counterpropagating case, the signal is now only observed where the $780 \mathrm{~nm}$ laser is at the correct frequency such that it can, in combination with the frequency-stabilized $776 \mathrm{~nm}$ laser, cause atoms to be excited to the $5 \mathrm{D}_{5 / 2}$ state. At the higher intensities studied we do observe a secondary resonance to the left of the main ${ }^{85} \mathrm{Rb}$ peak, but in this case the resonances are strongly power broadened and Stark shifted.

The data in Fig. 9 clearly illustrates that the spectroscopy method used is very sensitive to changes in position of the thin atomic medium. The observed modification of the spectra can be attributed to two main effects. Firstly, power broadening causes broader spectral features closer to the focus $(z=0 \mu \mathrm{m})$ due to the higher intensities experienced by the atoms. Secondly, moving away from the focus alters the amount of fluorescence generated by the atoms (via changing intensity and illuminated area) and also collected by the objective lens, causing the changes in count rates observed. Transit-time broadening due to the small spot size at the focus will also be a less significant contributing factor. The spectra that do not strongly exhibit the effects of power broadening (for example, those at $z= \pm 125 \mu \mathrm{m}$ ) also illustrate the benefit of our experimental scheme: narrow sub-Doppler fluorescence resonances are obtained in a copropagating geometry as well as the counterpropagating geometry seen in Fig. 8. This allows for a compact setup whereby a single objective delivers both excitation beams to a tightly focused spot in the atomic medium, as well as collecting the atomic fluorescence. In fact, a Voigt fit to the uppermost spectrum $(z=125 \mu \mathrm{m})$ yields a FWHM of $(57.5 \pm 0.6) \mathrm{MHz}$, the same order of magnitude as that achieved in the counterpropagating case.

The TPFM method in the copropagating geometry is also used to probe atomic vapor confined along a channel with a width and depth of $1 \mu \mathrm{m}$ [shown in Fig. 10(a)]. As displayed in Fig. 10(b), we find that a density gradient exists in the vapor as the fluorescence intensity reduces along the channel (with all other experimental parameters held constant between measurement sites). Given that this particular cell had been in operation for $1 \mathrm{yr}$, it would be reasonably expected that an effective steady state had been reached in terms of atomic vapor distribution. However, as is shown by the decaying integrated count rate, this is not the case. Instead, we have found that the atomic vapor density is reproducibly not uniform along channels with confinement on length scales less than or equal to $1 \mu \mathrm{m}$. The vapor pressure is perturbed simply by the presence of tight confinement, a result that provides important insight into the diffusion properties of atoms inside nanoscale structures. 

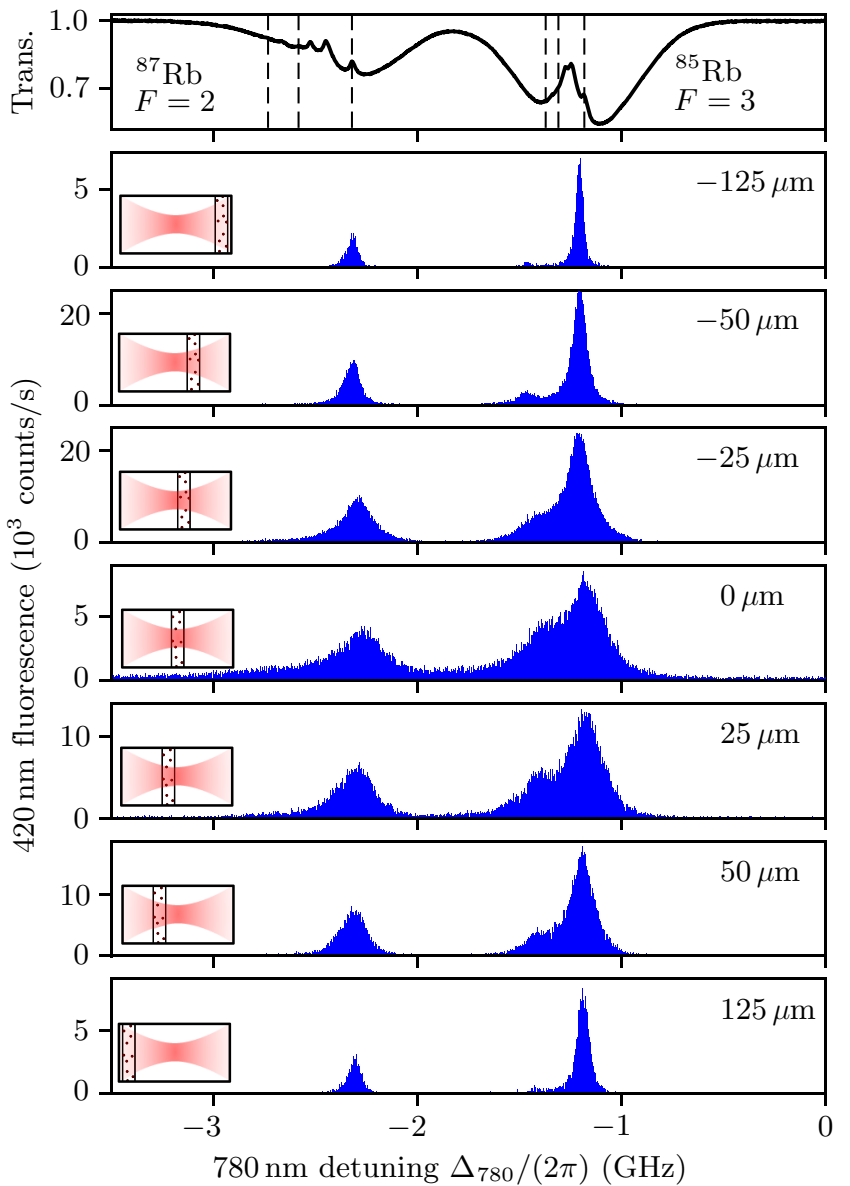

FIG. 9. Reference transmission (trans.) spectrum produced using a single-color pump-probe hyperfine spectroscopy setup [76] in a $75 \mathrm{~mm}$ cell. Each Doppler-broadened profile exhibits six sub-Doppler features (some too weak to be seen), with the three indicated corresponding to the hyperfine $5 \mathrm{P}_{3 / 2}$ states (see Fig. 8), and the other three to crossover resonances between these. Bottom: TPFM spectra recorded with $780 \mathrm{~nm}$ and $776 \mathrm{~nm}$ beams copropagating [both along path (a) in Fig. 7] incident on a region of the nanocell with $1 \mu \mathrm{m}$ depth in the direction of the excitation beams. The nanocell is translated with respect to the focal point of the beams (labeled $0 \mu \mathrm{m}$ ), such that the atoms experience different intensity environments at different $z$ positions (labeled). Inset diagrams illustrate this translation of the atomic layer with respect to the focus. High intensities at the focus give rise to power broadening, which in combination with the changing collection efficiency and illumination area, also results in the changing count rates between spectra. Features in the TPFM spectra relate to the hyperfine $5 \mathrm{P}_{3 / 2}$ states. Excitation beams have powers of $7 \mu \mathrm{W}$ at $780 \mathrm{~nm}$ and $20 \mu \mathrm{W}$ at $776 \mathrm{~nm}$, with a Rayleigh range of approximately $4 \mu \mathrm{m}$. Each dataset has an integration time of $1-10$ mins at a cell temperature of $65^{\circ} \mathrm{C}$.

For the experiments reported in Fig. 10, the excitation beam powers used are a compromise between signal strength and power broadening. Hence, by fitting Voigt profiles to the spectra we obtain an average FWHM of $140 \mathrm{MHz}$ and, as illustrated in Fig. 10(c), show that the
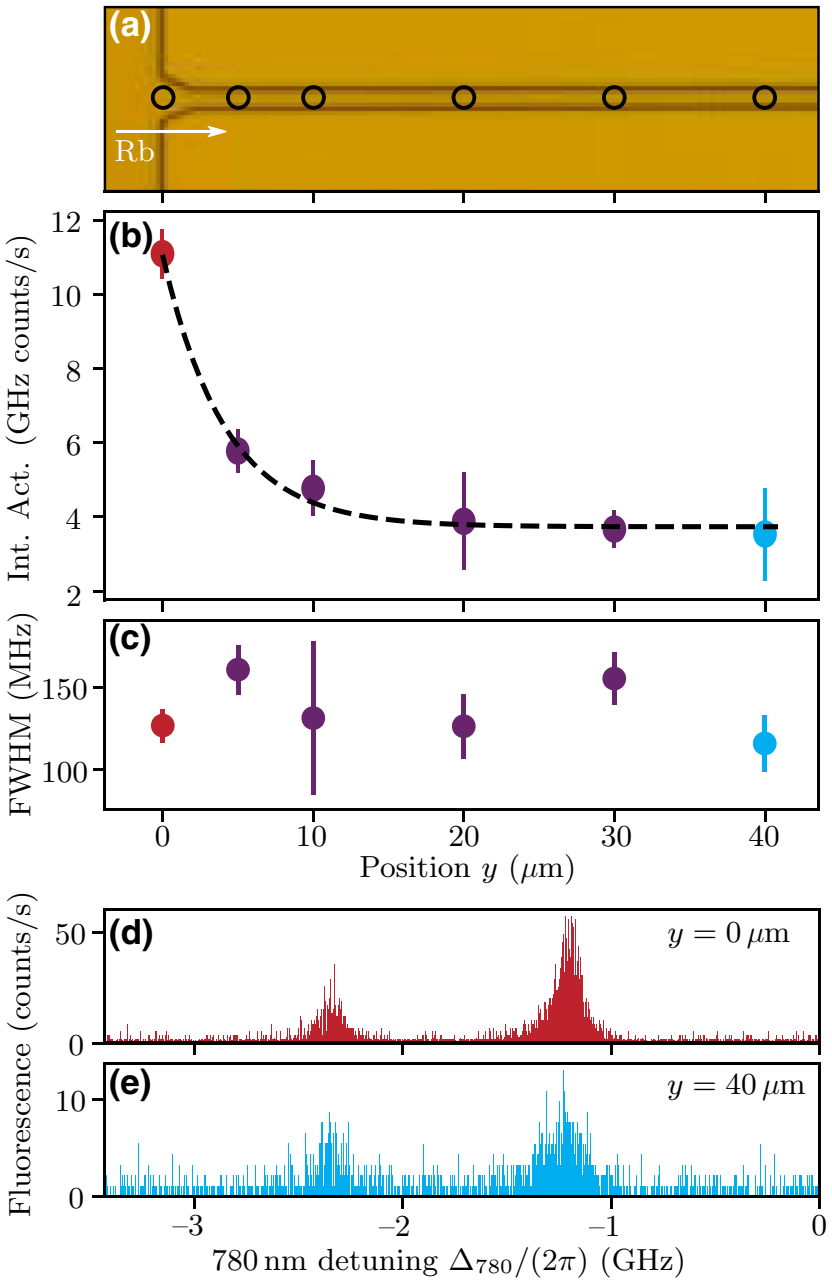

FIG. 10. (a) Bright-field image of a single channel, with a width and depth of $1 \mu \mathrm{m}$. In this image the source of rubidium atoms (reservoir) is from the left, as indicated. TPFM spectra are recorded at various locations along this channel (indicated). All spectra are empirically fitted with Voigt profiles, and from these fits the (b) integrated activity (Int. Act.) and (c) FWHMs are plotted as a function of position. An empirical exponential decay is fitted to the activity data (black dashed line), which yields a characteristic decay length of $(4 \pm 1) \mu \mathrm{m}$. Error bars for both the activity and FWHM values are estimated using the functional approach [77]. Panels (d) and (e) show two example spectra, color matched to the extreme points studied. This dataset is taken with powers of $50 \mathrm{nW}$ at 780 and $776 \mathrm{~nm}$, integration times of $0.5-9 \mathrm{~h}$, and a cell temperature of $60^{\circ} \mathrm{C}$.

FWHM does not vary significantly between sites in this tight confinement regime. The observed power broadening alludes to the tight focus of the beam that delivers a high intensity even at the low powers used. In Figs. 10(d) and $10(\mathrm{e})$, we present spectra corresponding to the closest and farthest measurement points, which have FWHMs of $(130 \pm 10)$ and $(120 \pm 20) \mathrm{MHz}$, respectively.

The TPFM method offers promise for the detection and study of low numbers of atoms confined to nanoscale 
structures. From the count rates observed in the spectrum shown in Fig. 10(e), we have extracted an estimate of the number of atoms excited to the $5 \mathrm{D}_{5 / 2}$ state. Accounting for the efficiency and throughput of our detection setup, as well as the lifetime of the $5 \mathrm{D}_{5 / 2}$ state, we estimate that the on-resonance count rate observed corresponds to a mean value of just 0.01 atoms excited to the $5 \mathrm{D}_{5 / 2}$ state within the detection volume. If instead we extract the atomic number density from the measured cell temperature and the rubidium vapor pressure curve, as is standard in experiments with bulk vapor cells [78], we find a mean ground state atom number of 1.5 within the same volume. Whilst we do not believe the bulk number density relationships to exactly hold in a thin cell (a consequence of tight confinement that our results in Fig. 10 begin to shed light on), this calculation provides an estimate for comparison. The extracted value of 1.5 ground state atoms is consistent with the experimental value of 0.01 atoms in the $5 \mathrm{D}_{5 / 2}$ state, as we would expect that only a fraction of atoms are excited to this state. Further modeling is, however, beyond the scope of this work.

Our data have also shown that it is possible to spectrally filter fluorescence signals from the TPFM method in such a way that the noise observed far off resonance, such as that in the spectra shown in Figs. 8, 10(d), and $10(\mathrm{e})$, is consistent with the dark count rate measured for the photon-counting PMT used in this work. This dark count rate is approximately less than or equal to $5 \mathrm{cps}$, and the input laser powers used in this work lie in the microwatt-nanowatt range. As such, this method is highly sensitive to low atom numbers (exemplified by the estimated atom number and moderate cell temperatures used in this section), whilst also allowing for long integration times limited only by dark noise. Thus, given our demonstrated experimental versatility and considerable control over atom numbers through cell temperature and confinement geometries, work towards interrogating low numbers of atoms is a promising avenue of further study.

\section{CONCLUSION}

We have designed and fabricated a nanostructured alkali-metal vapor cell that offers flexible optical access and high-NA imaging. Our glue-free design has proven to be durable and reliable, avoiding outgassing and degradation of the internal atmosphere over time. The formation of nanoscale structures inside our cell, via direct-write laser lithography and reactive ion etching, is flexible and highly customizable.

We have illustrated the versatility of optical access afforded by our nanocell design through the methods of TIRF and TPFM. The TIRF method allows for fine control of the atomic excitation region on the nanometer scale, enabling our study of the transit-time broadening induced on this length scale. Meanwhile, TPFM is a high signalto-noise dark-field measurement, and is used in this work to illustrate the potential of nanocells for sensing applications by studying the spectral responses of the vapor layer. Here our high-NA focusing also enables strong driving of even weaker transitions, and it is this effect, rather than Doppler broadening, that dominates the observed line shapes. Finally, using the TPFM method, we have spectroscopically probed atoms confined by $1 \mu \mathrm{m}$ in two dimensions. Through this method, we have demonstrated that tight confinement regimes modify the local atomic vapor density, paving the way for development towards room temperature atom-based devices with close control of the atom number at the level of single atoms.

The highly localized control of the atom number available by patterning confinement geometries that we have demonstrated opens doors towards the possibility of fewand single-atom applications. For example, the local vapor density could be controlled in such a way as to produce a single-atom source, for example by patterning nanochannel geometries to, on average, allow only a single atom from the thermal ensemble to pass through. This could open doors to heralded single-atom generation. Engineering local atomic density towards having only a single atom on average within an interaction region would then hold promise for work towards single-photon sources, for which our platform would provide a scalable and robust architecture.

The nanoscopic character of our vapor cell and the omnipresence of its walls readily provide sub-Doppler spectral resolution, giving access to individual hyperfine transitions of rubidium. Other technical advantages of our cells include the integrated ITO heating system and the high excitation and collection efficiency of our high-NA approach, which allow for operating the chip at relatively low temperatures compatible with biological sensing. The combination of these features with high-NA lateral resolution and the ultra-thin extent of the atoms makes our nanocell a powerful, spatially selective sensing tool. A special example of current interest in sensing is magnetometry with nanoscale resolution [18], and indeed sensitive magnetometry has recently been demonstrated using nanocells [15]. Our chip and measurement platform allows for improved spatial selectivity and high lateral resolution, especially for probing magnetic fields close to surfaces. The thin front panel and compactness of our design are key benefits in this regard, especially with regards to the ITO heater that removes the problem of bulky cell ovens experienced with previously reported vapor cells. That we can pattern arbitrary nanostructured geometries, rather than the standard wedge shape, also gives considerably greater versatility for extremely spatially selective measurements (e.g., spatially selective field imaging in one dimension using a thin atomic layer, or in two dimensions using a patterned nanochannel). The bespoke nature 
of our fabrication process also makes it possible, for example, to deposit material layers during cell manufacture that ultimately reside inside the finished cell. This could allow measurement of magnetic fields extremely close to material surfaces, enabled by the proximity of the atomic nanolayer.

The architecture of our nanocell makes it well adapted to the application of microscopy and spectroscopy techniques that are routine in nano-optics. As well as TIRF and TPFM, further potential examples include stimulated emission depletion fluorescence microscopy [79]; fluorescence correlation spectroscopy [80]; and interferometric scattering - a scheme that has previously been used to detect single nanoparticles and even charge transport via Rayleigh scattering $[69,81]$. These detection schemes, along with the versatility of the cell design and manufacturing process, offer access to a multitude of investigative routes. Further potential directions of study include the use of lightinduced atomic desorption to locally modify the atomic vapor density, depositing microelectrodes or micropatterning structures such as waveguides or microring resonators within the nanocells, or even adding dipole trapping to the setup [82]. Maturing the platform with integration of optical components to an on-chip design offers a promising route towards highly scalable atom-based quantum technologies.

The data presented in this paper are available from Durham Research Online [83].

\section{ACKNOWLEDGMENTS}

The authors are grateful to Dr. Sofia de Carvalho Ribeiro for editorial discussions. We also thank Benjamin Gmeiner and Pierre Türschmann for their contributions to the early phase of this work. We gratefully acknowledge support by the Max Planck Society and EPSRC under Grant No. EP/R002061/1.

T.F.C. and W.J.H. contributed equally to this work.

[1] S. Knappe, P. Schwindt, V. Shah, L. Hollberg, J. Kitching, L. Liew, and J. Moreland, A chip-scale atomic clock based on ${ }^{87} \mathrm{Rb}$ with improved frequency stability, Opt. Express 13, 1249 (2005).

[2] M. M. Boyd, A. D. Ludlow, S. Blatt, S. M. Foreman, T. Ido, T. Zelevinsky, and J. Ye, ${ }^{87} \mathrm{Sr}$ Lattice Clock with Inaccuracy below $10^{-15}$, Phys. Rev. Lett. 98, 083002 (2007).

[3] M. A. Zentile, J. Keaveney, L. Weller, D. J. Whiting, C. S. Adams, and I. G. Hughes, ElecSus: A program to calculate the electric susceptibility of an atomic ensemble, Comput. Phys. Commun. 189, 162 (2015).

[4] J. Keaveney, C. S. Adams, and I. G. Hughes, Elecsus: Extension to arbitrary geometry magneto-optics, Comput. Phys. Commun. 224, 311 (2018).
[5] J. Keaveney, S. A. Wrathmall, C. S. Adams, and I. G. Hughes, Optimized ultra-narrow atomic bandpass filters via magneto-optic rotation in an unconstrained geometry, Opt. Lett. 43, 4272 (2018).

[6] G. Bison, R. Wynands, and A. Weis, Dynamical mapping of the human cardiomagnetic field with a room-temperature, laser-optical sensor, Opt. Express 11, 904 (2003).

[7] P. D. D. Schwindt, S. Knappe, V. Shah, L. Hollberg, J. Kitching, L.-A. Liew, and J. Moreland, Chip-scale atomic magnetometer, Appl. Phys. Lett. 85, 6409 (2004).

[8] M. V. Balabas, D. Budker, J. Kitching, P. D. D. Schwindt, and J. E. Stalnaker, Magnetometry with millimeter-scale antirelaxation-coated alkali-metal vapor cells, J. Opt. Soc. Am. B 23, 1001 (2006).

[9] D. Budker and M. Romalis, Optical magnetometry, Nat. Phys. 3, 227 (2007).

[10] G. Bison, N. Castagna, A. Hofer, P. Knowles, J. L. Schenker, M. Kasprzak, H. Saudan, and A. Weis, A room temperature 19-channel magnetic field mapping device for cardiac signals, Appl. Phys. Lett. 95, 173701 (2009).

[11] R. Wyllie, M. Kauer, R. T. Wakai, and T. G. Walker, Optical magnetometer array for fetal magnetocardiography, Opt. Lett. 37, 2247 (2012).

[12] V. K. Shah and R. T. Wakai, A compact, high performance atomic magnetometer for biomedical applications, Phys. Med. Biol. 58, 8153 (2013).

[13] D. Sheng, S. Li, N. Dural, and M. V. Romalis, Subfemtotesla Scalar Atomic Magnetometry Using Multipass Cells, Phys. Rev. Lett. 110, 1 (2013).

[14] E. Boto, S. S. Meyer, V. Shah, O. Alem, S. Knappe, P. Kruger, T. M. Fromhold, M. Lim, P. M. Glover, P. G. Morris, R. Bowtell, G. R. Barnes, and M. J. Brookes, A new generation of magnetoencephalography: Room temperature measurements using optically-pumped magnetometers, Neuroimage 149, 404 (2017).

[15] E. Klinger, H. Azizbekyan, A. Sargsyan, C. Leroy, D. Sarkisyan, and A. Papoyan, Proof of the feasibility of a nanocell-based wide-range optical magnetometer, Appl. Opt. 59, 2231 (2020).

[16] J. Keaveney, W. J. Hamlyn, C. S. Adams, and I. G. Hughes, A single-mode external cavity diode laser using an intra-cavity atomic Faraday filter with short-term linewidth $<400 \mathrm{kHz}$ and long-term stability of $<1 \mathrm{MHz}$, Rev. Sci. Instrum. 87, 1 (2016).

[17] R. S. Mathew, F. Ponciano-Ojeda, J. Keaveney, D. J. Whiting, and I. G. Hughes, Simultaneous two-photon resonant optical laser locking (STROLLing) in the hyperfine Paschen-Back regime, Opt. Lett. 43, 4204 (2018).

[18] J. R. Maze, P. L. Stanwix, J. S. Hodges, S. Hong, J. M. Taylor, P. Cappellaro, L. Jiang, M. V. Gurudev Dutt, E. Togan, A. S. Zibrov, A. Yacoby, R. L. Walsworth, and M. D. Lukin, Nanoscale magnetic sensing with an individual electronic spin in diamond, Nature 455, 644 (2008).

[19] V. Sandoghdar, C. I. Sukenik, E. A. Hinds, and S. Haroche, Direct Measurement of the van der Waals Interaction between an Atom and its Images in a Micron-Sized Cavity, Phys. Rev. Lett. 68, 3432 (1992).

[20] C. I. Sukenik, M. G. Boshier, D. Cho, V. Sandoghdar, and E. A. Hinds, Measurement of the Casimir-Polder Force, Phys. Rev. Lett. 70, 560 (1993). 
[21] B. Zambon and G. Nienhuis, Reflection and transmission of light by thin vapor layers, Opt. Commun. 143, 308 (1997).

[22] J. Keaveney, A. Sargsyan, U. Krohn, I. G. Hughes, D. Sarkisyan, and C. S. Adams, Cooperative Lamb Shift in an Atomic Vapor Layer of Nanometer Thickness, Phys. Rev. Lett. 108, 173601 (2012).

[23] J. Petersen, J. Volz, and A. Rauschenbeutel, Chiral nanophotonic waveguide interface based on spin-orbit interaction of light, Science 346, 67 (2014).

[24] Fam Le Kien and A. Rauschenbeutel, Electromagnetically induced transparency for guided light in an atomic array outside an optical nanofiber, Phys. Rev. A 91, 053847 (2015).

[25] P. Lodahl, S. Mahmoodian, S. Stobbe, A. Rauschenbeutel, P. Schneeweiss, J. Volz, H. Pichler, and P. Zoller, Chiral quantum optics, Nature 541, 473 (2017).

[26] Fam Le Kien and A. Rauschenbeutel, Nanofiber-mediated chiral radiative coupling between two atoms, Phys. Rev. A 95, 023838 (2017).

[27] T. Peyrot, Y. R. P. Sortais, A. Browaeys, A. Sargsyan, D. Sarkisyan, J. Keaveney, I. G. Hughes, and C. S. Adams, Collective Lamb Shift of a Nanoscale Atomic Vapor Layer within a Sapphire Cavity, Phys. Rev. Lett. 120, 243401 (2018).

[28] S. Briaudeau, D. Bloch, and M. Ducloy, Sub-Doppler spectroscopy in a thin film of resonant vapor, Phys. Rev. A 59, 3723 (1999).

[29] D. Bloch and M. Ducloy, in Adv. At. Mol. Opt. Phys. (Academic Press, 2005), Vol. 50, p. 91.

[30] M. Fichet, G. Dutier, A. Yarovitsky, P. Todorov, I. Hamdi, I. Maurin, S. Saltiel, D. Sarkisyan, M.-P. Gorza, D. Bloch, and M. Ducloy, Exploring the van der Waals atom-surface attraction in the nanometric range, Europhys. Lett. 77, 54001 (2007).

[31] J. Kitching, S. Knappe, and E. A. Donley, Atomic sensors A review, IEEE Sens. J. 11, 1749 (2011).

[32] T. Peyrot, Ch. Beurthe, S. Coumar, M. Roulliay, K. Perronet, P. Bonnay, C. S. Adams, A. Browaeys, and Y. R. P. Sortais, Fabrication and characterization of super-polished wedged borosilicate nano-cells, Opt. Lett. 44, 1940 (2019).

[33] T. H. Sander, J. Preusser, R. Mhaskar, J. Kitching, L. Trahms, and S. Knappe, Magnetoencephalography with a chip-scale atomic magnetometer, Biomed. Opt. Express 3, 981 (2012).

[34] B. Lounis and M. Orrit, Single-photon sources, Rep. Prog. Phys. 68, 1129 (2005).

[35] L. Jiang, J. M. Taylor, and M. D. Lukin, Fast and robust approach to long-distance quantum communication with atomic ensembles, Phys. Rev. A 76, 012301 (2007).

[36] H. J. Kimble, The quantum internet, Nature 453, 1023 (2008).

[37] A. I. Lvovsky, B. C. Sanders, and W. Tittel, Optical quantum memory, Nat. Photonics 3, 706 (2009).

[38] F. Ripka, H. Kübler, R. Löw, and T. Pfau, A roomtemperature single-photon source based on strongly interacting Rydberg atoms, Science 362, 446 (2018).

[39] Y. Wang, J. Li, S. Zhang, K. Su, Y. Zhou, K. Liao, S. Du, H. Yan, and S. L. Zhu, Efficient quantum memory for singlephoton polarization qubits, Nat. Photonics 13, 346 (2019).
[40] J. Reichel, W. Hänsel, and T. W. Hänsch, Atomic Micromanipulation with Magnetic Surface Traps, Phys. Rev. Lett. 83, 3398 (1999).

[41] M. Keil, O. Amit, S. Zhou, D. Groswasser, Y. Japha, and R. Folman, Fifteen years of cold matter on the atom chip: Promise, realizations, and prospects, J. Mod. Opt. 63, 1840 (2016).

[42] E. A. Hinds and V. Sandoghdar, Cavity QED level shifts of simple atoms, Phys. Rev. A 43, 398 (1991).

[43] C. Henkel and V. Sandoghdar, Single-molecule spectroscopy near structured dielectrics, Opt. Commun. 158, 250 (1998).

[44] K. A. Whittaker, J. Keaveney, I. G. Hughes, A. Sargsyan, D. Sarkisyan, and C. S. Adams, Optical Response of GasPhase Atoms at Less Than $\lambda / 80$ from a Dielectric Surface, Phys. Rev. Lett. 112, 253201 (2014).

[45] T. Aoki, B. Dayan, E. Wilcut, W. P. Bowen, A. S. Parkins, T. J. Kippenberg, K. J. Vahala, and H. J. Kimble, Observation of strong coupling between one atom and a monolithic microresonator, Nature 443, 671 (2006).

[46] J. Marek, Observation of superradiance in $\mathrm{Rb}$ vapour, J. Phys. B 12, L229 (1979).

[47] G. Walker, A. S. Arnold, and S. Franke-Arnold, TransSpectral Orbital Angular Momentum Transfer via FourWave Mixing in Rb Vapor, Phys. Rev. Lett. 108, 243601 (2012).

[48] D. J. Whiting, R. S. Mathew, J. Keaveney, C. S. Adams, and I. G. Hughes, Four-wave mixing in a non-degenerate fourlevel diamond configuration in the hyperfine Paschen-Back regime, J. Mod. Opt. 65, 713 (2018).

[49] A. R. Bhagwat and A. L. Gaeta, Nonlinear optics in hollow-core photonic bandgap fibers, Opt. Express 16, 5035 (2008).

[50] D. Sarkisyan, D. Bloch, A. Papoyan, and M. Ducloy, SubDoppler spectroscopy by sub-micron thin Cs vapour layer, Opt. Commun. 200, 201 (2001).

[51] T. Baluktsian, C. Urban, T. Bublat, H. Giessen, R. Löw, and T. Pfau, Fabrication method for microscopic vapor cells for alkali atoms, Opt. Lett. 35, 1950 (2010).

[52] K. A. Whittaker, J. Keaveney, I. G. Hughes, A. Sargysyan, D. Sarkisyan, B. Gmeiner, V. Sandoghdar, and C. S. Adams, Interrogation and fabrication of $\mathrm{nm}$ scale hot alkali vapour cells, J. Phys. Conf. Ser. 635, 122006 (2015).

[53] L.-A. Liew, S. Knappe, J. Moreland, H. Robinson, L. Hollberg, and J. Kitching, Microfabricated alkali atom vapor cells, Appl. Phys. Lett. 84, 2694 (2004).

[54] S. Liron, D. G. Bopp, S. A. Schima, V. N. Maurice, and J. E. Kitching, Chip-scale atomic diffractive optical elements, Nat. Commun. 10, 3156 (2019).

[55] L. Stern, B. Desiatov, I. Goykhman, and U. Levy, Nanoscale light-matter interactions in atomic cladding waveguides, Nat. Commun. 4, 1548 (2013).

[56] L. Stern, R. Zektzer, N. Mazurski, and U. Levy, Enhanced light-vapor interactions and all optical switching in a chip scale micro-ring resonator coupled with atomic vapor, Laser Photon. Rev. 10, 1016 (2016).

[57] R. Ritter, N. Gruhler, W. H. P. Pernice, H. Kübler, T. Pfau, and R. Löw, Coupling thermal atomic vapor to an integrated ring resonator, New J. Phys. 18, 103031 (2016). 
[58] R. Ritter, N. Gruhler, H. Dobbertin, H. Kübler, S. Scheel, W. Pernice, T. Pfau, and R. Löw, Coupling Thermal Atomic Vapor to Slot Waveguides, Phys. Rev. X 8, 021032 (2018).

[59] L. Midolo, A. Schliesser, and A. Fiore, Nano-opto-electromechanical systems, Nat. Nanotechnol. 13, 11 (2018).

[60] A. Reiserer and G. Rempe, Cavity-based quantum networks with single atoms and optical photons, Rev. Mod. Phys. 87, 1379 (2015).

[61] U. D. Jentschura, M. Janke, and M. DeKieviet, Theory of noncontact friction for atom-surface interactions, Phys. Rev. A 94, 022510 (2016).

[62] B. Gmeiner, A. Maser, T. Utikal, S. Götzinger, and V. Sandoghdar, Spectroscopy and microscopy of single molecules in nanoscopic channels: Spectral behavior vs. confinement depth, Phys. Chem. Chem. Phys. 18, 19588 (2016).

[63] See Supplemental Material at http://link.aps.org/supple mental/10.1103/PhysRevApplied.14.034054 for a detailed description of cell thickness measurements and the integrated heating solution.

[64] C. S. Adams and I. G. Hughes, Optics 2 f: From Fourier to Fresnel (Oxford University Press, Oxford, 2018).

[65] J. Marek and P. Münster, Radiative lifetimes of excited states of rubidium up to quantum number $n=12$, J. Phys. B 13, 1731 (1980).

[66] D. Sheng, A. Pérez Galván, and L. A. Orozco, Lifetime measurements of the $5 d$ states of rubidium, Phys. Rev. A 78, 062506 (2008).

[67] A. Sargsyan, A. Papoyan, I. G. Hughes, C. S. Adams, and D. Sarkisyan, Selective reflection from an $\mathrm{Rb}$ layer with a thickness below $\lambda / 12$ and applications, Opt. Lett. 42, 1476 (2017).

[68] J. P. Woerdman and M. F. H. Schuurmans, Spectral narrowing of selective reflection from sodium vapour, Opt. Commun. 14, 248 (1975).

[69] R. W. Taylor and V. Sandoghdar, Interferometric scattering microscopy: Seeing single nanoparticles and molecules via Rayleigh scattering, Nano Lett. 19, 4827 (2019).

[70] P. Siddons, C. S. Adams, C. Ge, and I. G. Hughes, Absolute absorption on rubidium D lines: Comparison between theory and experiment, J. Phys. B 41, 155004 (2008).

[71] D. Axelrod, T. P. Burghardt, and N. L. Thompson, Total internal reflection fluorescence, Annu. Rev. Biophys. Bioeng. 13, 247 (1984).
[72] U. Volz and H. Schmoranzer, Precision lifetime measurements on alkali atoms and on helium by beam-gas-laser spectroscopy, Phys. Scripta T65, 48 (1996).

[73] W. Demtröder, Laser Spectroscopy (Springer, Berlin, Heidelberg, 2008).

[74] D. J. Whiting, J. Keaveney, C. S. Adams, and I. G. Hughes, Direct measurement of excited-state dipole matrix elements using electromagnetically induced transparency in the hyperfine paschen-back regime, Phys. Rev. A 93, 043854 (2016).

[75] C. Carr, C. S. Adams, and K. J. Weatherill, Polarization spectroscopy of an excited state transition, Opt. Lett. 37, 118 (2012).

[76] D. A. Smith and I. G. Hughes, The role of hyperfine pumping in multilevel systems exhibiting saturated absorption, Am. J. Phys. 72, 631 (2004).

[77] I. G. Hughes and T. P. A. Hase, Measurements and Their Uncertainties: A Practical Guide to Modern Error Analysis (Oxford University Press, Oxford, 2010).

[78] C. B. Alcock, V. P. Itkin, and M. K. Horrigan, Vapour pressure equations for the metallic elements: $298-2500 \mathrm{~K}$, Can. Metall. Q. 23, 309 (1984).

[79] S. W. Hell and J. Wichmann, Breaking the diffraction resolution limit by stimulated emission: Stimulated-emissiondepletion fluorescence microscopy, Opt. Lett. 19, 780 (1994).

[80] E. L. Elson and D. Magde, Fluorescence correlation spectroscopy. I. Conceptual basis and theory, Biopolymers 13, 1 (1974).

[81] M. Delor, H. L. Weaver, Q. Yu, and N. S. Ginsberg, Imaging material functionality through three-dimensional nanoscale tracking of energy flow, Nat. Mat. 19, 56 (2019).

[82] V. Sandoghdar, C. I. Sukenik, S. Haroche, and E. A. Hinds, Spectroscopy of atoms confined to the single node of a standing wave in a parallel-plate cavity, Phys. Rev. A 53, 1919 (1996).

[83] T. F. Cutler, W. J. Hamlyn, J. Renger, K. A. Whittaker, D. Pizzey, I. G. Hughes, V. Sandoghdar, and C. S. Adams, Data for: "Nanostructured Alkali-Metal Vapor Cells," Durham Research Online, https://doi.org/10.15128/r1g732 d901w (2020). 